\title{
Thermal conductivity of indium arsenide nanowires with wurtzite and zinc blende phases
}

\author{
Feng Zhou, ${ }^{1}$ Arden L. Moore, ${ }^{2}$ Jessica Bolinsson, ${ }^{3}$ Ann Persson, ${ }^{3,4}$ Linus Fröberg, ${ }^{3}$ Michael T. Pettes, ${ }^{2}$ Huijun Kong, ${ }^{2}$ \\ Lew Rabenberg, ${ }^{1,2}$ Philippe Caroff, ${ }^{5}$ Derek A. Stewart, ${ }^{6}$ Natalio Mingo ${ }^{7,8}$ Kimberly A. Dick, ${ }^{3,9}$ Lars Samuelson, ${ }^{3}$ \\ Heiner Linke, ${ }^{3}$ and $\mathrm{Li} \mathrm{Shi}^{1,2, *}$ \\ ${ }^{1}$ Materials Science and Engineering Program, The University of Texas at Austin, Austin, Texas 78712, USA \\ ${ }^{2}$ Department of Mechanical Engineering, The University of Texas at Austin, Austin, Texas 78712, USA \\ ${ }^{3}$ Solid State Physics, Lund University, Box 118, S-22100 Lund, Sweden \\ ${ }^{4}$ Physics Department and Materials Science Institute, University of Oregon, Eugene, Oregon 97403, USA \\ ${ }^{5}$ Institut d'Electronique, de Microélectronique et de Nanotechnologie, UMR CNRS 8520, Avenue Poincaré, B.P. 60069, F-59652 Villeneuve \\ d'Ascq, France \\ ${ }^{6}$ Cornell Nanoscale Facility, Cornell University, Ithaca, New York 14850, USA \\ ${ }^{7}$ CEA, LITEN, F-38054 Grenoble, France \\ ${ }^{8}$ University of California at Santa Cruz, Jack Baskin School of Engineering, Santa Cruz, California 95064, USA \\ ${ }^{9}$ Polymer \& Materials Chemistry, Box 124, Lund University, S-22100 Lund, Sweden
}

(Received 1 November 2010; revised manuscript received 18 March 2011; published 19 May 2011)

\begin{abstract}
The thermal conductivity of wurtzite and zinc blende indium arsenide nanowires was measured using a microfabricated device, with the crystal structure of each sample controlled during growth and determined by transmission electron microscopy. Nanowires of both phases showed a reduction of the thermal conductivity by a factor of 2 or more compared to values reported for zinc blende indium arsenide bulk crystals within the measured temperature range. Theoretical models were developed to analyze the measurement results and determine the effect of phase on phonon transport. Branch-specific phonon dispersion data within the discretized first Brillouin zone were calculated from first principles and used in numerical models of volumetric heat capacity and thermal conductivity. The combined results of the experimental and theoretical studies suggest that wurtzite indium arsenide possesses similar volumetric heat capacity, weighted average group velocity, weighted average phonon-phonon scattering mean free path, and anharmonic scattering-limited thermal conductivity as the zinc blende phase. Hence, we attribute the differing thermal conductivity values observed in the indium arsenide nanowires of different phases to differences in the surface scattering mean free paths between the nanowire samples.
\end{abstract}

DOI: 10.1103/PhysRevB.83.205416

PACS number(s): 65.80.-g, 44.10.+i, 65.40.Ba

\section{INTRODUCTION}

Indium arsenide (InAs) is a small-band-gap $(0.35 \mathrm{eV})$ semiconductor that is widely used in diode lasers and infrared detectors. ${ }^{1-4}$ Additionally, the electron mobility of InAs is high due to its small electron effective mass. Bulk InAs crystals typically exhibit a cubic zinc blende (ZB) structure. However, the growth of wurtzite (WZ) phase InAs nanowires (NWs) has been demonstrated using chemical beam epitaxy $(\mathrm{CBE})^{5,6}$ and other techniques. ${ }^{7}$ The synthesis of these WZ phase InAs NWs resulted in extensive interest within the research community to determine how their electrical and thermal transport properties compare to those of ZB InAs NWs. The phase-specific transport properties as well as their size scaling behaviors are relevant to both nanoelectronic and thermoelectric energy conversion applications. In terms of thermoelectric energy conversion, nanostructuring has been actively investigated as an approach to reducing the phonon thermal conductivity and increasing the power factor. ${ }^{8}$ Motivated by these potential applications, the electronic band structure of WZ InAs has been calculated, ${ }^{9,10}$ while electron transport measurements of WZ InAs NWs have also been reported. ${ }^{11-15}$ However, while a significant amount of theoretical and experimental work has been focused on investigating the electrical properties of these NWs, comparatively little effort has been made to analyze thermal transport in WZ phase InAs NWs. ${ }^{16}$
There are two and four atoms per primitive unit cell in the $\mathrm{ZB}$ and $\mathrm{WZ}$ phase, respectively. As the number of atoms and size of the primitive cell increase, additional zone boundaries are introduced in the reciprocal space in an extended zone representation. In the folded zone representation, the size of the Brillouin zone is reduced and additional opticalphonon branches are introduced with increasing numbers of atoms in the unit cell. If the masses or bonding differ substantially for different atoms in each unit cell, the new zone boundaries can lead to energy gaps in the dispersion curve and reduced group velocities near the zone boundaries and for the additional optical branches. For the case in which the thermal conductivity contribution of the additional optical branches becomes negligible due to a small group velocity, Slack identified a general trend of decreasing thermal conductivity with increasing numbers of atoms in a unit cell. ${ }^{17,18}$

Moreover, the additional zone boundaries tend to increase the loci of scattered phonon wave vector states that participate in three-phonon scattering. However, coherence can be lost in the Fourier transform of the different bonds in each cell as the number of atoms in the cell increases. ${ }^{19}$ This effect can reduce the matrix elements of the three-phonon processes. For a random distribution of atoms in each cell, Roufosse and Klemens showed that the anharmonic scattering rate is substantially independent of the number 
of atoms per cell because the two effects cancel each other. $^{19}$

For ZB crystals, there are a total of six phonon branches, including three acoustic modes and three optical modes. In comparison, the WZ phase has twelve phonon branches comprised of three acoustic, three low-lying optical, and six highly placed optical branches. The loci of scattered phonon wave vector states are increased by three-phonon processes involving phonons from the acoustic and the low-lying optical branches. Acoustic-optical scattering and four-phonon processes were used by Steigmeier and co-workers to explain the temperature dependence of their measured thermal conductivity of III-V compounds. ${ }^{20-22}$ On the other hand, Ecsedy and Klemens found that four-phonon processes are much weaker than three-phonon processes even at temperatures of up to $1000 \mathrm{~K}$, and that the relative impact of cell size on these scattering processes is not inherently clear. ${ }^{23}$

Among bulk semiconductors, silicon carbide ( $\mathrm{SiC}$ ), gallium nitride $(\mathrm{GaN})$, and aluminum nitride $(\mathrm{AlN})$ exist in both $\mathrm{ZB}$ and $\mathrm{WZ}$ phases. For AIN and GaN, the phonon dispersion relations for both phases have been reported. ${ }^{24,25}$ For AlN, one study using molecular dynamics simulation found a two to five times higher thermal conductivity for the WZ phase than the ZB phase. ${ }^{26}$ However, another work calculated a fourfold longer average phonon mean free path in ZB AlN than in WZ AlN due to increased optical-phonon scattering in the WZ phase. ${ }^{25}$ We have found reported experimental thermal conductivity data for only the WZ phase of bulk AlN and GaN crystals, with no data being found for the $\mathrm{ZB}$ phase of these respective materials. In comparison, experimental results for both $\mathrm{ZB} 3 \mathrm{C}-\mathrm{SiC}$ and hexagonal $4 \mathrm{H}-\mathrm{SiC}$ and $6 \mathrm{H}-\mathrm{SiC}$ polytypes of $\mathrm{SiC}$ show almost identical thermal conductivity values for temperatures above $200 \mathrm{~K} .^{27-29}$ When combined with other results in the literature, ${ }^{30}$ the data suggest that the thermal conductivity of $\mathrm{SiC}$ is more sensitive to impurity content and defect concentration than phase.

Given the different effects of unit cell size reported for AlN and $\mathrm{SiC}$, the role of phase on the thermal conductivity of InAs has remained elusive. In particular, it remains to be investigated whether the phonon group velocity near the zone boundaries for the WZ phase would be substantially reduced and whether the effect of additional acoustic-optical scattering processes is more pronounced than reduced three-phonon matrix elements for the particular atomic arrangement in the WZ unit cell of InAs NWs.

In this work, we report the thermal conductivity measurement of both WZ phase and ZB phase InAs NWs using suspended micro-thermometer devices. Since the WZ phase of bulk InAs has not yet been synthesized, there are no experimental data on the phonon dispersion for this phase. We have used density functional theory to calculate the equilibrium crystal structures and phonon dispersions for both WZ and ZB phases of InAs from first principles. The $a b$ initio phonon dispersions are used in a numerical thermal conductivity model to analyze the measurement data. The analysis shows that the volumetric heat capacity, the weighted average group velocity, the average phonon-phonon scattering rate, and the anharmonic scattering-limited thermal conductivity are similar between the $\mathrm{WZ}$ and $\mathrm{ZB}$ phases for the case of InAs, and that diffuse surface scattering is the main cause of the considerably lower measured thermal conductivity of InAs NWs of both phases compared to the literature values for ZB bulk InAs crystals.

\section{EXPERIMENTAL METHODS AND RESULTS}

InAs NWs with WZ phase were grown by CBE. Details of the WZ InAs NW growth parameters used here can be found in the literature. ${ }^{5,6}$ The NWs grown using this method were single crystal and the diameter of the NW was determined by the gold seed particle size. ${ }^{5,6}$ A representative scanning electron microscopy (SEM) image of the as-grown WZ phase NW array is shown in Fig. 1(a), while a representative SEM image of as-grown ZB NWs is shown in Fig. 2(a). Details of the ZB InAs NW growth can also be found in the literature. ${ }^{31}$ These ZB NWs were grown by metal-organic vapor phase epitaxy and have a tapered shape due to the increased lateral overgrowth on ZB surfaces. ${ }^{31}$ It has been shown that a substantial amount of background carbon impurities can be incorporated during radial overgrowth on InAs NWs. ${ }^{15}$ The radially grown material on the NW sides is therefore expected to be $n$-doped compared to the interior.

A micro-thermometer device such as the one shown in Fig. 1(b) was used to measure the thermal conductivity of each of the individual NW samples. The device incorporated two adjacent silicon nitride $\left(\mathrm{SiN}_{X}\right)$ membranes each suspended with six $\mathrm{SiN}_{X}$ beams. ${ }^{32,33}$ One serpentine platinum resistance thermometer (PRT) and two platinum electrodes were patterned on each membrane. A through-substrate hole directly under the suspended membranes allows for transmission
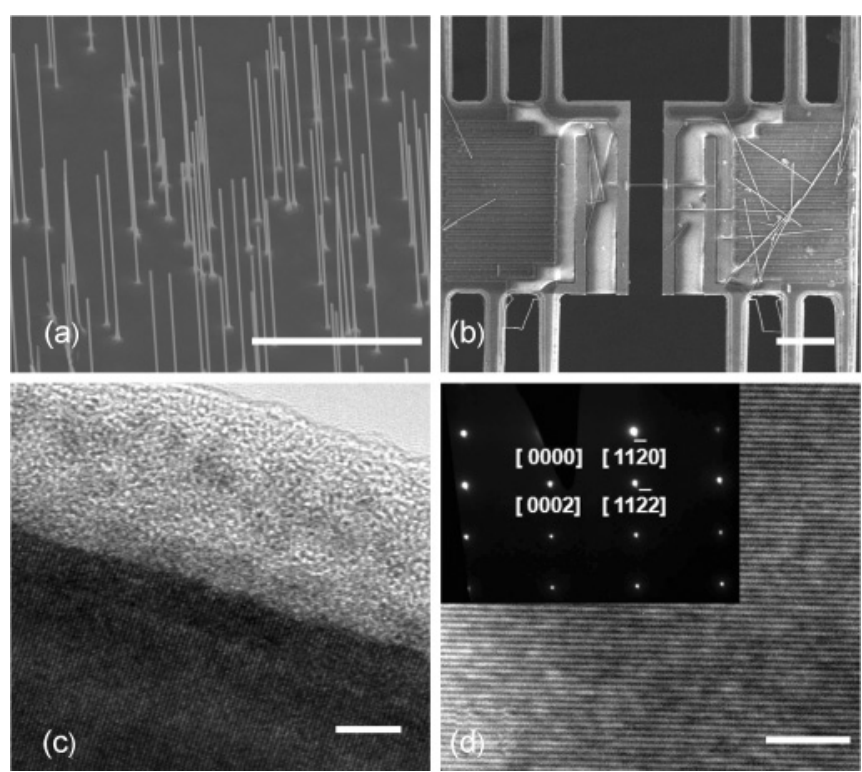

FIG. 1. (a) SEM image of the as-grown WZ phase InAs NW arrays. (b) SEM image of WZ phase sample NW4 trapped between the two suspended micro-thermometers of the measurement device. (c) TEM image of NW4 showing an amorphous shell and the crystalline NW core. (d) High-resolution TEM showing the lattice fringes of the InAs NW core. The inset is the corresponding diffraction pattern taken with a beam spot size of $1.5 \mu \mathrm{m}$. Scale bars are $5 \mu \mathrm{m}$ for (a) and (b) and $5 \mathrm{~nm}$ for (c) and (d). 

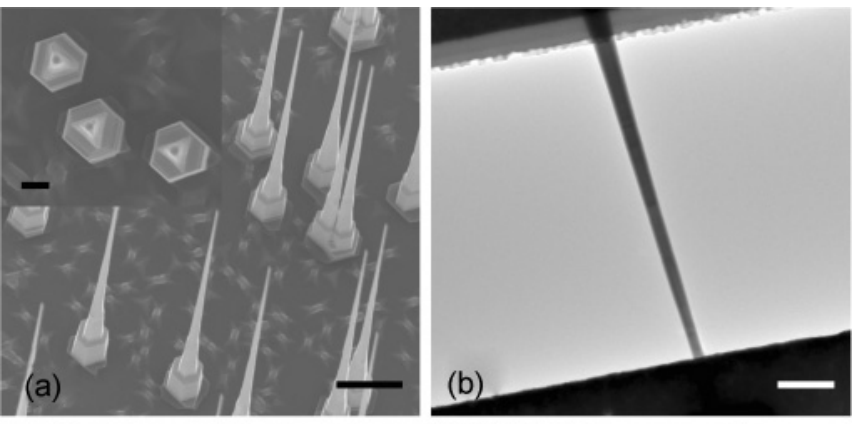

(c)
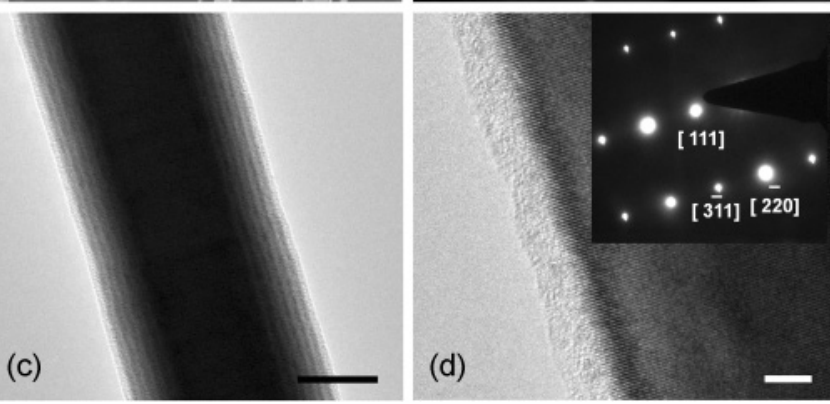

FIG. 2. (a) Tilted side-view SEM image of the as-grown ZB phase InAs NW arrays showing the tapered shape. The inset is a top-down SEM image showing the triangular cross section of the NWs grown from a hexagonal base. (b) Low magnification TEM image of NW2 showing the tapered shape. (c) Low magnification TEM image of NW2 showing the thickness fringes at the NW edges caused by the triangular cross section. (d) High-resolution TEM image of NW2. The inset is the corresponding diffraction pattern taken with a beam spot size of $1.5 \mu \mathrm{m}$. Scale bars are $1 \mu \mathrm{m}, 200 \mathrm{~nm}, 0.5 \mu \mathrm{m}, 50 \mathrm{~nm}$, and $5 \mathrm{~nm}$ for (a), the inset in (a), (b), (c), and (d), respectively.

electron microscopy (TEM) analysis of each individual NW sample after the thermal measurement was completed.

Table I summarizes the lateral dimension of the crystalline core, amorphous layer thickness, phase, and growth direction for each of the measured NWs. The WZ phase NWs form a hexagonal cross section. ${ }^{11} \mathrm{~A}$ representative high-resolution (HR) TEM image for the WZ phase sample NW4 is shown in Fig. 1(c). The amorphous layer surrounding the crystalline core of NW4 is thicker than the values shown in Table I for other NWs and is likely oxide formed during sample preparation, measurement, or before TEM images were obtained following measurement. From the HRTEM image and the corresponding diffraction pattern in Fig. 1(d), the growth direction of NW4 was determined to be along the $c$ axis, i.e., the $\langle 0001\rangle$ direction. The growth directions of the other WZ phase NWs were also found to be along the $\langle 0001\rangle$ direction, consistent with previous observations of as-grown WZ phase InAs NWs. ${ }^{6,34}$

Figure 2 is the collection of the SEM and TEM images of the ZB phase NW2. Figure 2(a) is the tilted side-view SEM image of the as-grown $\mathrm{ZB}$ phase InAs NW arrays showing their tapered shape, while the inset is a top-down SEM image revealing the triangular cross section of the NWs grown from a hexagonal base. Due to the triangular cross section of the $\mathrm{ZB}$ NW samples, the lateral dimensions in Table I are the widths of one edge of the triangular cross section of the crystalline core of the NWs. NW2 and NW3 were tapered along the suspended segment such that the two lateral dimension values in Table I refer to the dimensions of the crystalline core measured at the ends of the suspended NW segment where the respective NW meets the membranes of the microdevice. NW1 showed no appreciable tapering in comparison. The tapered shape of the ZB NW2 is also illustrated in the low-magnification TEM image in Fig. 2(b). The periodic thickness fringes at the NW edges in the low magnification TEM image of NW2 [Fig. 2(c)] are attributed to the triangular cross section of the $\mathrm{ZB}$ phase NWs. The HRTEM image of NW2 and the corresponding diffraction pattern (inset) show that the ZB phase NW2 was grown along the $\langle 111\rangle$ direction. The other two $\mathrm{ZB}$ phase NWs were also found to be along the $\langle 111\rangle$ direction, which is consistent with the observations of previous work on as-grown ZB phase InAs NWs. ${ }^{34}$

No twins or stacking faults were observed within the NW regions imaged using TEM for either the ZB or WZ NWs. For the ZB NWs, the presence of a significant number of defects would be observable even in SEM images as their presence would change the cross section due to threefold symmetry. For the WZ NWs, it would be necessary to tilt the samples to the $\langle 110\rangle$ axis to observe stacking faults, whereas most of the TEM images available for the WZ samples in this work are oriented to $\langle 112\rangle$ such that any stacking faults are not visible. In previous work, WZ NWs grown by CBE typically have stacking fault density in the range of one to three faults per micrometer of length for all growth conditions. ${ }^{15}$ Based on these statistics, few stacking faults are expected to be present over the suspended NW lengths of $\sim 3-4 \mu \mathrm{m}$ for the WZ NWs.

Because of residual gas molecules in the evacuated sample space, radiation, and substrate heating, a small background thermal conductance signal $\left(G_{\mathrm{bg}}\right)$ was measured using a blank device without a NW bridging the two membranes. This background signal was subtracted from the measured sample thermal conductance of the device with the NW

TABLE I. Summary of the measured InAs NWs and TEM measurements. For the tapered NW samples NW2 and NW3, the two lateral dimension values refer to the dimensions of the crystalline core measured at the ends of the suspended NW segment. The remaining NWs had uniform diameter along their suspended length.

\begin{tabular}{lccccc}
\hline \hline Label & Lateral dimension $(\mathrm{nm})$ & Amorphous layer thickness $(\mathrm{nm})$ & Phase & Growth Direction & Cross section \\
\hline NW1 & 148 & 3 & Zinc blende & $\langle 111\rangle$ & Triangular \\
NW2 & $97-179$ & 4 & Zinc blende & $\langle 111\rangle$ & Triangular \\
NW3 & $69-121$ & 8 & Zinc blende & $\langle 111\rangle$ & Triangular \\
NW4 & 66 & 11 & Wurtzite & $\langle 0001\rangle$ & Hexagonal \\
NW5 & 63 & 9 & Wurtzite & $\langle 0001\rangle$ & Hexagonal \\
\hline \hline
\end{tabular}


sample to obtain the thermal conductance of the NW including contributions from both the crystalline core and the amorphous shell. The thermal conductance of the amorphous layer was calculated as $G_{a}=\kappa_{a} A_{\text {shell }} / L$, where the length $L$ and cross sectional area $A_{\text {shell }}$ are based on the dimensions determined by TEM, and $\kappa_{a}$ is the thermal conductivity of the amorphous shell. The amorphous shell is most likely either surface oxide, amorphous carbon deposited from electron beam exposure, or a combination of the two materials. Using the composition of oxides on InAs surfaces found in the literature, ${ }^{35}$ the temperature-dependent thermal conductivity of the oxide was calculated from a minimum thermal conductivity model ${ }^{36}$ to be in the range of $0.4-0.6 \mathrm{~W} / \mathrm{m} \mathrm{K}$ at $300 \mathrm{~K}$. From the reported thermal conductivity data of amorphous carbon films of different densities ${ }^{37}$ and the reported density of amorphous carbon from electron beam-induced deposition, ${ }^{38}$ the thermal conductivity of the amorphous carbon was determined to be approximately $0.2 \mathrm{~W} / \mathrm{m} \mathrm{K}$. Hence, $\kappa_{a}$ was taken to be between 0.2 and $0.6 \mathrm{~W} / \mathrm{m} \mathrm{K}$. Based on this range, the amorphous shell contribution to the NW thermal conductance was less than $10 \%$ throughout the measured temperature range for all samples. The thermal conductance of the crystalline core of the NW was obtained as $G_{\mathrm{NW}}=G_{\text {measured }}-G_{\mathrm{bg}}-G_{a}$, which was at least ten times larger than $G_{\mathrm{bg}}$ and $G_{a}$.

The thermal conductivity of the NWs with uniform cross section was calculated as $\kappa=G_{\mathrm{NW}} L / A_{c}$, where $L$ is the length of the suspended NW segment and $A_{c}$ is the crosssectional area calculated using the lateral dimension of the NW. For the WZ NWs with hexagonal cross section, the axisymmetric rotational orientation of the cross section could not be determined from the obtained top-view SEM or TEM images. Hence, the lateral dimension of the crystalline core measured by electron microscopy could correspond to the corner-to-corner or edge-to-edge length of the hexagonal cross section or be for some orientation of the hexagonal cross section in between these two limits. Here, an average value of $A_{c}$ between these two limits was used to calculate $\kappa$ for the WZ NWs, with the uncertainty in $A_{c}$ given by the two $A_{c}$ limits propagated into the associated values of $\kappa$. For the tapered NWs with an equilateral triangle cross section, the thermal conductivity was calculated based on a similar analysis in Ref. 39 as

$$
\kappa=\frac{4 G_{\mathrm{NW}} L\left(\frac{1}{W_{1}}-\frac{1}{W_{2}}\right)}{\sqrt{3}\left(W_{2}-W_{1}\right)}
$$

where the two lateral dimensions $W_{1}$ and $W_{2}$ refer to the dimensions measured at the two ends of the suspended NW segment, as given in Table I.

As shown in Fig. 3, the measured thermal conductivity values of the $\mathrm{WZ}$ and $\mathrm{ZB} \mathrm{NW}$ samples are well below the reported thermal conductivity of bulk ZB InAs. ${ }^{40,41}$ In addition, the thermal conductivity values of the WZ NWs were found to be lower than those of the ZB NWs. While the measurement results showed a markedly lower thermal conductivity for the WZ phase InAs NWs, there are several factors besides the phase difference that could impact the NW thermal conductivity values. For example, the WZ NWs are of smaller cross section than the ZB NWs. This may result in increased phonon boundary scattering and a reduced thermal

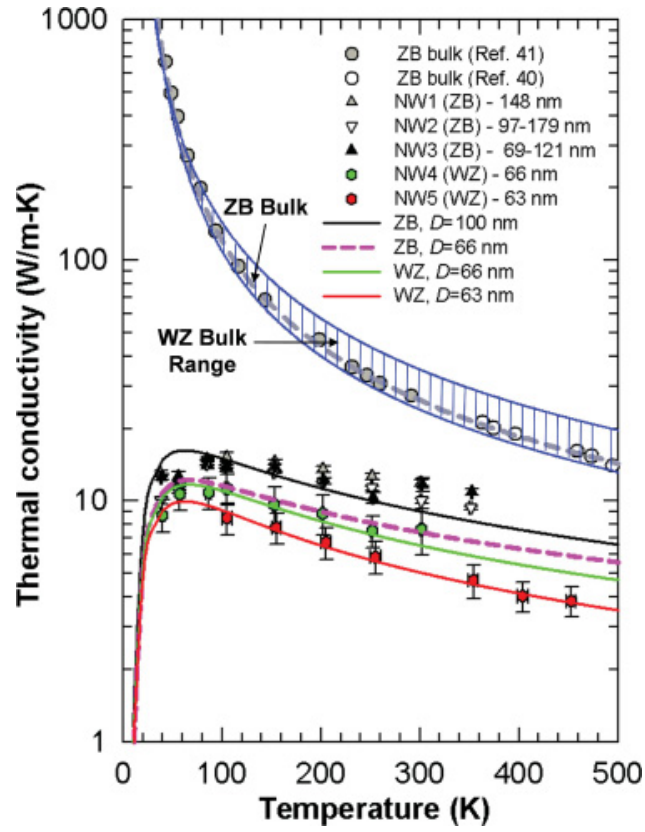

FIG. 3. (Color online) Thermal conductivity of the measured InAs NWs (symbols). Data for bulk ZB InAs (Refs. 40 and 41) are also shown. Calculation results for WZ and ZB InAs in both bulk and NW form are plotted as lines. The NW calculation results are shown for various boundary scattering mean free path $(D)$ values as discussed in Sec. III. For the tapered samples NW2 and NW3, the two values in the legend refer to the dimensions measured at the ends of the suspended NW segment.

conductivity. It should be noted that this difference in cross section size is likely due to radial overgrowth as the tip diameter of the two phases was similar.

\section{THEORETICAL ANALYSIS}

\section{A. Model description}

To separate the effects of different phases and different cross sectional areas on the measured thermal conductivity, we have developed a numerical model to analyze the measurement results. Analytical Callaway-type thermal conductivity models ${ }^{42,43}$ in which the Brillouin zone is approximated as a sphere have been widely used to calculate the thermal conductivity of Si and other cubic phase materials. Although this spherical Brillouin zone approximation may be acceptable for ZB InAs [Fig. 4(a)], the Brillouin zone of WZ InAs is a hexagonal prism for which the spherical approximation can potentially severely overestimate or underestimate the phonon density of states at high frequencies depending on the chosen cutoff wave vector, as shown in Fig. 4(b). Hence, a numerical thermal conductivity model that does not rely on the spherical approximation was developed in this work. This model uses branch-specific phonon frequency data calculated at discrete wave vector points within the Brillouin zone of $\mathrm{ZB}$ and WZ InAs crystals. Because the lateral dimensions of the NWs are much larger than the dominant phonon wavelength within the measured temperature range, the phonon dispersions of the NWs are taken to be the same as that of the bulk crystal of the same phase. 

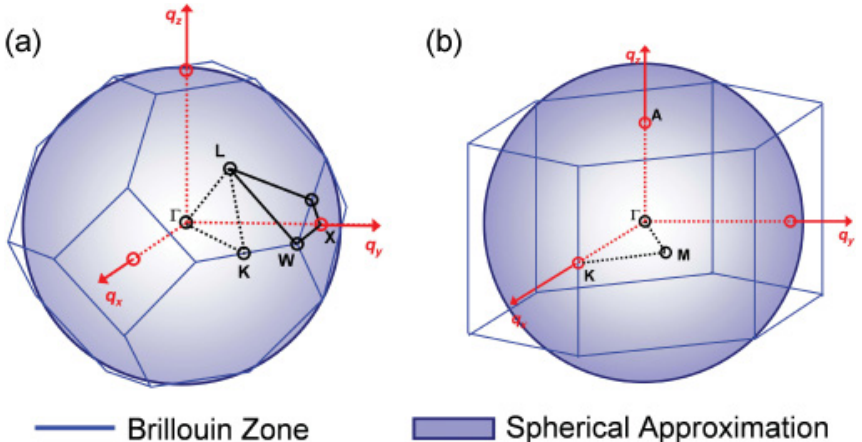

FIG. 4. (Color online) Comparison of the actual Brillouin zone of (a) ZB and (b) WZ InAs with a spherical Brillouin zone approximation.

The $a b$ initio phonon dispersions of the $\mathrm{ZB}$ and $\mathrm{WZ}$ crystal phases of InAs were calculated using density functional perturbation theory (DFPT) ${ }^{44}$ as implemented in the plane-wave pseudopotential code QUANTUM ESPRESSO ${ }^{45}$ The minimum total energy of each crystal was first determined by adjusting the crystal lattice constants and atomic positions based on Hellmann-Feynman forces. The local density approximation (LDA) and a plane-wave cutoff of 80 Ryd were used in all calculations. The In and As ions were described using Bachelet-Hamann-Schlueter ${ }^{46}$ (BHS) norm-conserving pseudopotentials available in the QUANTUM ESPRESSO distribution. ${ }^{47}$ Brillouin zone integration with a Monkhorst-Pack $k$-point grid of $16 \times 16 \times 16$ was used for the electronic properties of both crystal structures. The predicted lattice constant for ZB InAs was $a=5.96830 \AA$. In the case of WZ InAs, the relaxed crystal coordinates were $a=4.21086 \AA$, $c / a=1.641214$, and $u=0.374692$. The $\mathrm{ZB}$ phase was found to be more stable than the WZ phase by $6.28 \mathrm{meV} /$ atom.

Using DFPT, the interatomic force constants were directly calculated on a $6 \times 6 \times 6$ Monkhorst-Pack phonon $q$-vector grid in the Brillouin zone for both ZB and WZ InAs. Once these terms have been calculated, the phonon frequencies and eigenvectors can be determined for an arbitrary phonon $q$ vector through Fourier interpolation. The calculated phonon dispersions along the principal axes are shown in Fig. 5 along with the experimental data of Orlova ${ }^{48}$ for ZB InAs as determined by $x$-ray thermal diffuse scattering. The calculated phonon dispersion for bulk ZB InAs is also in good agreement with the prior ab initio results of Eckl et al. ${ }^{1}$ For the numerical model used in this work, the first Brillouin zone is discretized into a three-dimensional grid of phonon wave vector points. For ZB InAs, $q$ vectors were sampled on a $20 \times 20 \times$ 40 grid over a parallelepiped constructed from the three reciprocal lattice vectors. For WZ InAs, the phonon branches were sampled with a dense grid $(10 \times 10 \times 50)$ over a $1 / 6$ portion of the total Brillouin zone, which can be expanded to the full Brillouin zone via symmetry. It should be noted that particular care is required to ensure that phonon frequencies are matched with the proper phonon branches due to the occurrence of multiple branch crossings in the Brillouin zone. In this work, the phonon branches at a given $q$ vector are sorted by computing overlap matrices between eigenvectors at adjacent points in the Brillouin zone using an approach developed by Refson. ${ }^{49}$ Phonon eigenvectors at neighboring $q$ points with high overlap are taken to be part of the same branch and sorted accordingly. This approach works well in most cases given that a sufficiently dense mesh is used in the $q$ direction of interest.

Based on the calculated phonon dispersions in Fig. 5, the group velocity for the acoustic branches is very similar for both phases. This result is in agreement with the experimental finding that the speed of sound in WZ InAs NWs is comparable to that of bulk ZB InAs. ${ }^{50}$ In addition, the increased number of atoms in the WZ unit cell does not lead to energy gaps at the new zone boundaries because of identical atomic bonds in each unit cell.

The branch-specific frequency data obtained from the discretized Brillouin zone calculations were used to calculate the volumetric heat capacity $(C)$ of both $\mathrm{ZB}$ and $\mathrm{WZ}$ InAs according to

$$
C=\sum_{s} \sum_{q} \frac{\Delta q^{3} k_{B}}{8 \pi^{3}} \frac{x^{2} e^{x}}{\left(e^{x}-1\right)^{2}}, \quad x=\frac{\hbar \omega}{k_{B} T},
$$

where $T$ is the absolute temperature, $\hbar$ is the reduced Planck's constant, $k_{B}$ is the Boltzmann constant, and $\Delta q^{3}$ is the volume of each cell within the discretized Brillouin zone and is different between an interior cell and one on an edge or corner of the Brillouin zone. The summation is over different phonon branches $(s)$ and wave vectors $(q)$. As shown in Fig. 6(a), the calculation results for WZ and ZB InAs are almost identical, with both curves in good agreement with the reported experimental data for bulk ZB InAs. ${ }^{51,52}$ Similarly, AlShaikhi and Srivastava ${ }^{53}$ calculated nearly identical specific heat between the ZB and WZ phases of GaN and AlN at all but extremely low temperatures.

In addition to $C$, we have also calculated the $C v_{z}$ and $C v_{z}^{2}$ product from the discrete phonon frequencies as

and

$$
C v_{z}=\sum_{s} \sum_{q} \frac{\Delta q^{3} k_{B}}{8 \pi^{3}} v_{z} \frac{x^{2} e^{x}}{\left(e^{x}-1\right)^{2}}
$$

$$
C v_{z}^{2}=\sum_{s} \sum_{q} \frac{\Delta q^{3} k_{B}}{8 \pi^{3}} v_{z}^{2} \frac{x^{2} e^{x}}{\left(e^{x}-1\right)^{2}},
$$

where $v_{z}$ is the frequency-dependent phonon group velocity component along the direction of the temperature gradient for InAs NWs of the two different phases. As shown in Figs. 6(b) and 6(c), the as-calculated $C v_{z}$ is very similar for the two phases, whereas the $C v_{z}^{2}$ product is up to $6 \%$ higher for the WZ phase than the ZB phase. In addition, the $C v_{z} / C$ ratio is the weighted average group velocity, which is similar for both phases. Because the simplified kinetic theory yields $\kappa=$ $C v_{z} l_{\text {avg }}=C v_{z}^{2} \tau_{\text {avg }}$, where $l_{\text {avg }}$ and $\tau_{\text {avg }}$ are the average phonon scattering mean free path and time, ${ }^{54}$ respectively, the rather different thermal conductivity values measured for the InAs NWs of the two different phases are attributed to different $l_{\text {avg }}$ and $\tau_{\text {avg }}$ values for different NWs. 

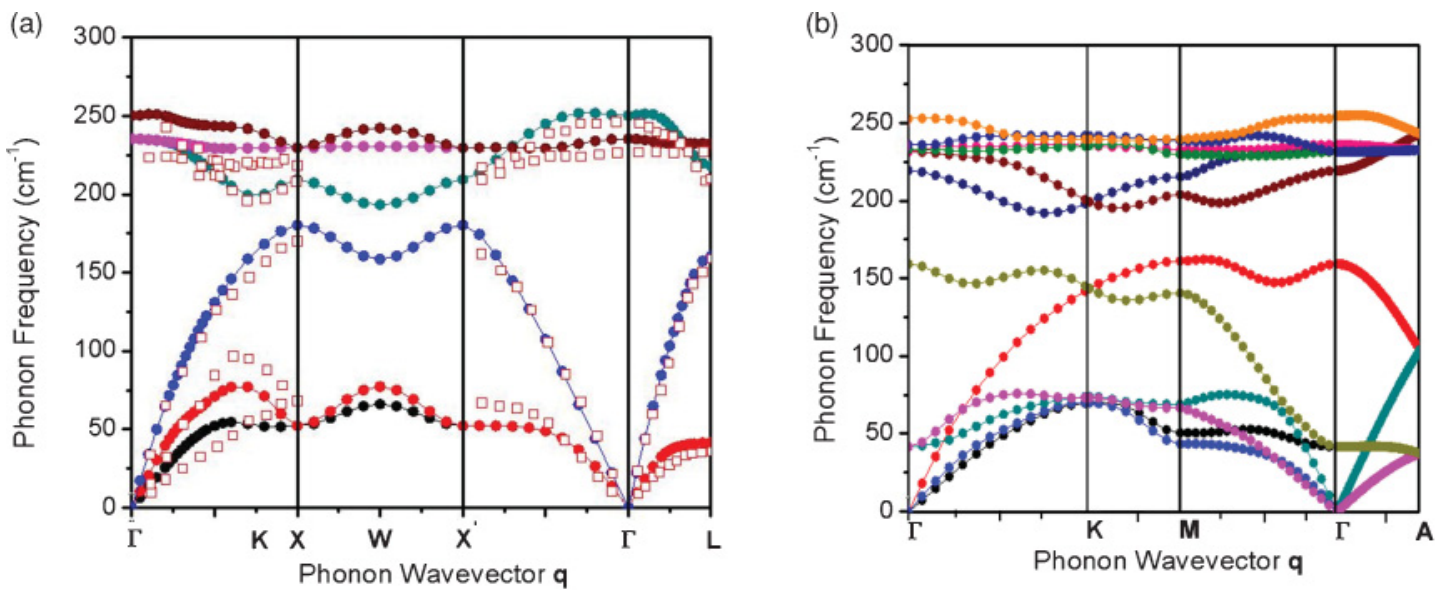

(c)
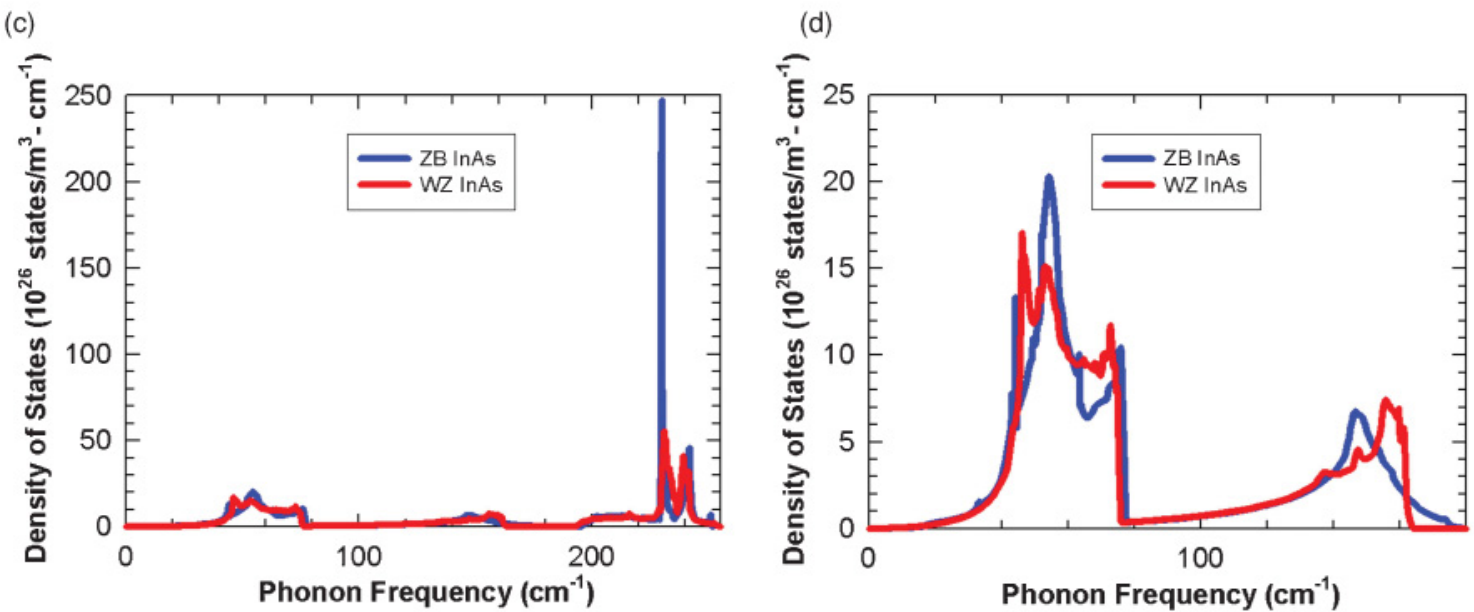

FIG. 5. (Color online) Phonon dispersions of (a) ZB and (b) WZ bulk InAs, where color is used to distinguish between phonon branches. Calculation results from this work in (a) and (b) are solid circles, while the experimental data for ZB InAs from the literature ${ }^{48}$ in (a) are open squares. The calculated phonon density of states for ZB and WZ InAs are shown in (c), with a rescaled view of the acoustic phonon region shown in (d) for clarity.

Instead of using the simplified kinetic theory, we calculate the thermal conductivity $(\kappa)$ from the discrete phonon dispersion data according to

$$
\kappa=\sum_{s} \sum_{q} \frac{\Delta q^{3} k_{B}}{8 \pi^{3}} v_{z}^{2} \tau \frac{x^{2} e^{x}}{\left(e^{x}-1\right)^{2}},
$$

where $\tau$ is the frequency-dependent relaxation time. According to Matthiessen's rule,

$$
\tau^{-1}=\tau_{i}^{-1}+\tau_{p-p, s}^{-1}+\tau_{b}^{-1},
$$

where $\tau_{i}^{-1}=A \omega^{4}, \quad \tau_{p-p, s}^{-1}=B_{s} \omega^{2} T \exp \left(-C_{s} / 3 T\right)$, and $\tau_{b}^{-1}=v / D$ are the scattering rate due to point defects, phononphonon, and boundary scattering processes, respectively, with the coefficients $A$ being sample-specific, $B_{s}$ and $C_{s}$ being material-specific, $D$ being the boundary scattering mean free path, and $v$ being the phonon group velocity. ${ }^{42,43,55-57}$ Here, $v$ instead of $v_{z}$ is used in the conversion between $\tau_{b}^{-1}$ and $D$ for each phonon mode because phonons are incident on the NW boundary from all crystallographic directions within the NW interior. The boundary scattering mean free path $D$ is typically related to the characteristic length $L_{c}$ by $D=\alpha L_{c}$. For an isotropic material, the value of the $\alpha$ term depends on cross section shape and surface specularity $p$, which ranges from 0 for the Casimir limit of a fully diffuse surface to unity for totally specular reflection. Thus, for a circular cross section, $\alpha=(1+p) /(1-p),{ }^{58}$ such that $D=d(\alpha=1)$ for fully diffuse surfaces, where $d$ is the diameter. Similarly, for a square cross section with diffuse surfaces, $D=1.12 L_{c}(\alpha=1.12)$, and for an equilateral triangle with diffuse surfaces, $D=0.714 L_{c}$ ( $\alpha=0.714)$, where $L_{c}$ is the side length of the square ${ }^{58}$ or equilateral triangle ${ }^{59}$ cross section, respectively. However, in materials with anisotropic sound velocities, phonon focusing effects can cause $\alpha$ to deviate from the respective isotropic values. ${ }^{60}$ This phenomenon was first studied in silicon, for which the branch-specific sound velocities differ by as much as $20 \%$ between the $\langle 100\rangle$ and $\langle 111\rangle$ directions. The experimental $\alpha$ values were found to be $19 \%$ lower for the $\langle 111\rangle$ direction and about $22 \%$ higher for the $\langle 100\rangle$ direction than the expected $\alpha$ value for isotropic materials. By comparison, $\mathrm{ZB}$ and WZ InAs have nearly the same degree of anisotropy in its branch-specific sound velocities as observed in silicon, suggesting that a similar degree of phonon focusing is possible. 
(a)

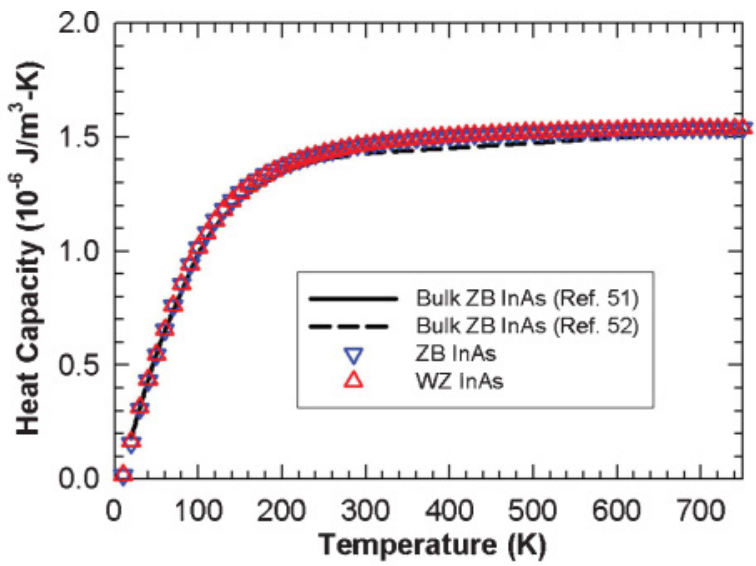

(b)

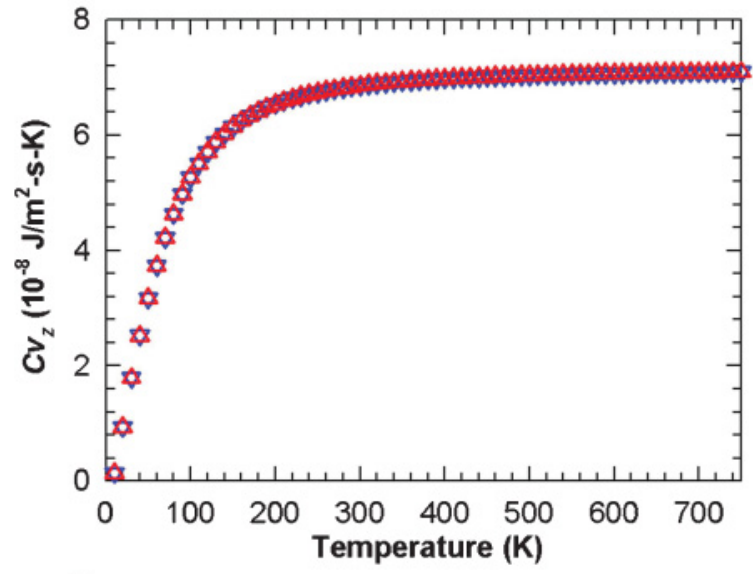

(c)

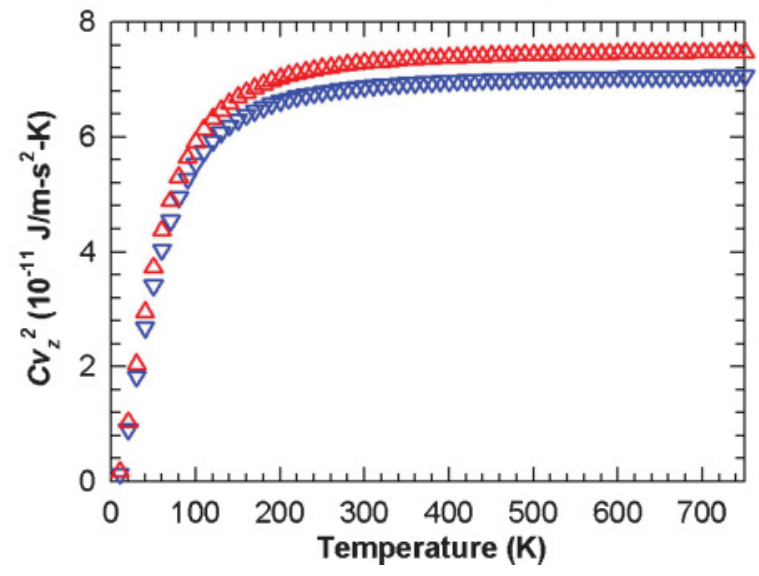

FIG. 6. (Color online) (a) Volumetric heat capacity, (b) $C v_{z}$ product, and (c) $C v_{z}^{2}$ product of $\mathrm{ZB}$ and WZ InAs crystals calculated with the use of the discretized dispersion data in the full Brillouin zone in comparison with experimental heat capacity data of bulk ZB InAs. ${ }^{51,52}$

\section{B. Zinc blende InAs}

For ZB phase bulk crystals, the coefficients $A, B_{s}$, and $C_{s}$ can be obtained by fitting the thermal conductivity model to the experimental bulk data. Although optical phonons make a large contribution to the calculated $C$ for both phases at temperature higher than $60 \mathrm{~K}$, their contribution to the thermal conductivity is negligible for the $\mathrm{ZB}$ phase because of their small $v_{z}$. Hence, optical branches are not included in Eq. (4) for the thermal conductivity calculation for the $\mathrm{ZB}$ phase. In this case, the reported experimental thermal conductivity of bulk $\mathrm{ZB}$ crystals can be fit with Eqs. (4) and (5) using $A=7 \times 10^{-46} \mathrm{~s}^{3}, B_{L}=0.27 \times$ $10^{-18} \mathrm{~s} / \mathrm{K}, B_{T}=2.8 \times 10^{-18} \mathrm{~s} / \mathrm{K}, C_{L}=230.6 \mathrm{~K}, C_{T}=$ $59.7 \mathrm{~K}$, and $D=2 \mathrm{~mm}$, where the subscripts $L$ and $T$ denote longitudinal and transverse acoustic phonon branches, respectively. The values for the phonon-phonon scattering coefficients obtained from fitting are quite similar to those obtained using the expressions given by Morelli et al. ${ }^{43}$ for umklapp processes. To ensure that the contribution from optical branches was indeed negligible for the ZB phase, a simple optical phonon-phonon scattering model ${ }^{61}$ based on the Klemens' channel of optical-phonon interactions ${ }^{62}$ was used to estimate their relative contribution to the total thermal conductivity. As expected, the optical- phonon contribution was less than $5 \%$ of the total thermal conductivity. Thus, to minimize the number of adjustable parameters within the model, the optical branches were excluded from modeling of ZB phase InAs NWs and bulk crystals.

It should be noted that while optical branches do not contribute directly to thermal transport due to their low group velocity, they provide important scattering channels for acoustic modes and thereby limit the thermal conductivity, as has been demonstrated by first-principles calculations of diamond, silicon, and germanium. ${ }^{63,64}$ However, including these processes directly and explicitly would introduce additional fitting parameters in the model. Instead, the above phenomenological phonon-phonon scattering model is used here to encompass all phonon-phonon processes without distinction between normal, umklapp, and optical scattering. Despite the use of the analytical umklapp phonon-phonon scattering expression for simplicity, by fitting experimental data the effects of phonon-phonon scattering events that include normal processes or optical branches are implicitly included in the model.

For modeling the ZB NW thermal conductivity, the phononphonon scattering coefficients found from fitting the bulk $\mathrm{ZB}$ thermal conductivity data were retained while the point defect scattering parameter $A$ and the boundary scattering mean free path $D$ were allowed to differ from their respective bulk ZB InAs values. The obtained boundary scattering mean free paths were $D=105 \mathrm{~nm}$ for NW1 and $D \sim 90-$ $100 \mathrm{~nm}$ for NW2 and NW3. For the observed NW surface roughness of $\sim 0.5-1 \mathrm{~nm}$ [Figs. 1 (c) and 2(d)] and an estimated dominant phonon wavelength on the order of $1 \mathrm{~nm}$ for InAs for the temperature range of this measurement, Ziman's surface specularity relation predicts diffuse phonon surface scattering. ${ }^{58}$ The obtained boundary scattering mean-free-path values $D$ obtained for the triangular $\mathrm{ZB}$ NWs are within $30 \%$ of the theoretical value given by $D=0.714 \mathrm{~W}$ for the isotropic case in the Casimir or diffuse surface limit, where $W$ is the edge length of the equilateral triangle cross section. ${ }^{59}$ Thus, the obtained mean-free-path values are close to the diffuse surface limit. With the phonon-phonon and boundary scattering parameters established, the point defect coefficient $A$ was adjusted to best fit the measured ZB NW thermal conductivity data. The adjustment of $A$ had little effect on the mid- to high-temperature thermal conductivity where phonon-phonon scattering processes dominate point defect scattering, but it improved the fit at low temperatures where 
point defect scattering is important. The results of this fitting are shown by the representative solid black line in Fig. 3 with $D=100 \mathrm{~nm}$ and $A=5 \times 10^{-44} \mathrm{~s}^{3}$.

\section{Wurtzite InAs}

Bulk thermal conductivity data for WZ InAs are unavailable for use in obtaining the associated phonon-phonon scattering coefficients. Thus, a different approach from that used in the modeling of the ZB phase was required for the $\mathrm{WZ}$ phase. The boundary scattering mean free path $D$ was varied from $\mathrm{NW}$ diameter $d$ up to several millimeters. Using the same $A$ value as found for $\mathrm{ZB}$ phase bulk InAs, for each value of $D$ the phononphonon scattering coefficients were adjusted to best fit the measured thermal conductivity data of the two WZ NWs using Eqs. (4) and (5) along with the calculated discrete Brillouin zone phonon dispersion data of the $\mathrm{WZ}$ phase. In addition to the acoustic branches, the three low-lying optical branches were included in the thermal conductivity calculation due to their non-negligible velocities in the WZ phase. The phononphonon scattering expressions and coefficients for these lowlying optical phonons are taken as the same as those for the acoustic branches of the same polarization.

It was found that for $D>\sim 1.5 d$, exceptionally strong phonon-phonon scattering was required to match the magnitude of the high-temperature $\mathrm{WZ}$ data. This causes a much stronger temperature dependence than observed in the experimental data and, as a result, the fit to the experimental data is poor. This discrepancy indicates that the WZ NW surface is close to being entirely diffuse similar to that found for the ZB NWs, and in agreement with Ziman's roughnessspecularity relation ${ }^{58}$ using the WZ NW surface roughness observed in TEM images. For $D \approx 1.5 d$, the fitting of the measurement data with different phonon-phonon scattering parameters provided moderate agreement from $\sim 200 \mathrm{~K}$ and above. However, for this case the peak in $\kappa$ at low temperatures was much higher than the measurement data such that $\sim 10$ times stronger point defect scattering than found for the $\mathrm{ZB}$ NWs was required to match the data. As stated previously, of the two phases, the ZB NWs are more likely to have carbon or other point defects introduced during growth, making the case of significantly stronger point defect scattering in the WZ NWs unlikely. By contrast, using $D \approx d$ provided a superior fit of the measurement data over the entire measured temperature range and a more physically realistic point defect scattering coefficient $A=1 \times 10^{-44} \mathrm{~s}^{3}$ between the $7 \times 10^{-46}$ and $5 \times$ $10^{-44} \mathrm{~s}^{3}$ values found for bulk ZB crystals reported in Ref. 41 and the ZB NWs studied here, respectively.

Setting $D=d$ for the WZ NWs, the phonon-phonon scattering parameters were adjusted to obtain the best fit to the WZ NW data at $\sim 200 \mathrm{~K}$ and above, where phononphonon scattering should dominate point defect scattering. This procedure yielded a set of phonon-phonon coefficients $B_{L}=(0.38-0.58) \times 10^{-18} \mathrm{~s} / \mathrm{K}, \quad B_{T}=(3.9-5.4) \times$ $10^{-18} \mathrm{~s} / \mathrm{K}, C_{L}=230.6 \mathrm{~K}$, and $C_{T}=59.7 \mathrm{~K}$, with the range of values dictated by the independent fittings of the data for NW4 and NW5, respectively. Here, fitting the experimental data in Fig. 3 for NW5 with $63 \mathrm{~nm}$ diameter gives the upper limit of the phonon-phonon scattering parameters, while fitting the data for NW4 with $66 \mathrm{~nm}$ diameter gives the lower limit. The obtained coefficients $B_{L}$ and $B_{T}$ are $48 \%-93 \%$ higher for the WZ phase than for the ZB phase. However, when the obtained phonon-phonon scattering coefficients for each phase are used to calculate the weighted average phonon-phonon scattering mean free path $l_{p-p}$ and mean free time $\tau_{p-p}$ using

$$
l_{p-p}=\frac{\sum_{s} \sum_{q} \Delta q^{3} v_{z}^{2} \tau_{p-p, s} \frac{x^{2} e^{x}}{\left(e^{x}-1\right)^{2}}}{\sum_{s} \sum_{q} \Delta q^{3} v_{z} \frac{x^{2} e^{x}}{\left(e^{x}-1\right)^{2}}}
$$

and

$$
\tau_{p-p}=\frac{\sum_{s} \sum_{q} \Delta q^{3} v_{z}^{2} \tau_{p-p, s} \frac{x^{2} e^{x}}{\left(e^{x}-1\right)^{2}}}{\sum_{s} \sum_{q} \Delta q^{3} v_{z}^{2} \frac{x^{2} e^{x}}{\left(e^{x}-1\right)^{2}}},
$$

the calculated $l_{p-p}$ and $\tau_{p-p}$ for the WZ phase range from comparable to $30 \%$ higher than the corresponding values for the ZB phase at intermediate and high temperatures. This result may be due to different Brillouin zones for the two phases, and in particular higher and lower density of states in the 45-52 and $52-60 \mathrm{~cm}^{-1}$ phonon frequency ranges, respectively, for the WZ phase than for the ZB phase, as shown in Figs. 4(c) and $4(d)$.

\section{Phase dependence of the thermal conductivity}

Using the obtained range of phonon-phonon scattering coefficients, $D=2 \mathrm{~mm}$, and $A=7 \times 10^{-46} \mathrm{~s}^{3}$, the range of anharmonic scattering-limited bulk thermal conductivity for the WZ phase was calculated to be similar to the bulk $\mathrm{ZB}$ thermal conductivity as shown in Fig. 3. Hence, this result suggests that bulk WZ InAs would not possess a much shorter phonon-phonon scattering mean free path or much lower thermal conductivity than bulk ZB InAs, similar to the comparable thermal conductivity demonstrated between the cubic and hexagonal polytypes of $\mathrm{SiC} .^{27,28}$

In addition, if the boundary scattering mean free path is set to be $D=66 \mathrm{~nm}$, the model based on the bulk phononphonon and defect scattering coefficients and discretized phonon dispersion data for the ZB phase yields a thermal conductivity curve that is rather similar to the measured thermal conductivity data for the WZ NW with $66 \mathrm{~nm}$ diameter (Fig. 3). With $D=66 \mathrm{~nm}$, the thermal conductivity is expected to be close to $\kappa=C v_{z} D$ because boundary scattering is comparable to or stronger than the phonon-phonon scattering and point defect scattering rates given by the values found from fitting the bulk ZB InAs data. Hence, the similarity between the numerically calculated $\mathrm{ZB}$ thermal conductivity with $D=$ $66 \mathrm{~nm}$ and the measured data for the WZ NW with $66 \mathrm{~nm}$ diameter verifies that both $\mathrm{ZB}$ and $\mathrm{WZ}$ InAs indeed possess similar $C v_{z}$ values, in agreement with the above calculation using the discrete Brillouin zone data for both phases [Fig. 6(b)].

The comparable thermal conductivity values found for the two phases may be related to the details of the respective crystal structures. The main crystalline difference between ZB phase NWs with $\langle 111\rangle$ growth direction and WZ phase NWs with $\langle 0001\rangle$ growth direction is the stacking order of the atomic layers, with $A B A B$ stacking in $\langle 0001\rangle$ WZ NWs and $A B C A B C$ growth in the case of $\langle 111\rangle \mathrm{ZB}$ NWs. In both stacking 
configurations, atoms are tetrahedrally bonded, and for the 12 second-nearest neighbors the overall atomic arrangement is very similar. For example, Nusimovici and Birman calculated the phonon dispersion for both ideal $\mathrm{WZ}$ and $\mathrm{ZB}$ cadmium sulfide $(\mathrm{CdS}) .{ }^{65}$ Upon unfolding the $\mathrm{WZ}(\Gamma-A)$ phonon branch and comparing it with the $\mathrm{ZB}(\Gamma-L)$ line, they found that $\mathrm{WZ}$ and ZB CdS had identical TA branches and that the ZB LA phonon branch had slightly higher frequencies. This close match in phonon branches along the unfolded WZ $(\Gamma-A)$ and $\mathrm{ZB}(\Gamma-L)$ directions could also explain why the measured sound velocities for WZ InAs NWs were so similar to bulk ZB InAs. ${ }^{50}$

Besides similar phonon dispersions, the similar average phonon-phonon scattering rates found for both phases suggest that the effect of additional acoustic-optical scattering may be canceled by the reduced coherence in the Fourier transform of the different bonds of the increased WZ unit cell size. According to Roufosse and Klemens, ${ }^{19}$ the matrix elements of the three-phonon scattering processes contain the factor $\sum_{\vec{r}} e^{-i \vec{b} \cdot \vec{r}}$, where the summation is over all atoms in one primitive cell, $\vec{r}$ is the position of an atom, and $\vec{b}$ is the reciprocal lattice vector involved in the umklapp process. We calculated that this factor for the WZ phase is about 1.9, 1.4, and 0 times that for the $\mathrm{ZB}$ phase for the three reciprocal lattice vectors. Because these values are less than the factor of 2 difference in the number of atoms in the primitive cell for the two phases, the increased number of atoms in the WZ unit cell indeed results in the loss of coherence that tends to cancel the effect of additional acoustic-phonon scattering.

In addition, the $c / a$ ratio of $\mathrm{WZ}$ InAs was found from first principles to be only $0.5 \%$ greater than the ideal ratio of $\sqrt{8 / 3}$. Similarly, the $\mathrm{WZ}$ form of $\mathrm{SiC}, 2 \mathrm{H}-\mathrm{SiC}$, has a $c / a$ ratio of $1.641,{ }^{1}$ which is $0.4 \%$ greater than the ideal $c / a$ ratio, and has been shown experimentally to have comparable thermal conductivity to cubic SiC. ${ }^{66}$ In contrast, the WZ phase of AlN is more closely packed in the $\langle 0001\rangle$ direction with a $c / a$ of $1.600,{ }^{1}$ roughly $\sim 2 \%$ less than the ideal ratio. This atomic configuration with a $c / a$ ratio far from the ideal ratio may lead to a different thermal conductivity of the $\mathrm{WZ}$ phase than the $\mathrm{ZB}$ phase. Further work on the thermal conductivity of materials of varying $c / a$ ratios in the $\mathrm{WZ}$ phase is needed to elucidate what role the packing of layers has on thermal conductivity in relation to that of the $\mathrm{ZB}$ phase.

\section{CONCLUSION}

In this work, the thermal conductivity of $\mathrm{WZ}$ and $\mathrm{ZB}$ NWs was measured using a microfabricated device. The obtained values for both phases demonstrated a reduction of the thermal conductivity by a factor of 2 or more compared to bulk ZB InAs within the measured temperature range. A numerical model based on ab initio WZ InAs phonon dispersion data was developed to account for the nonspherical Brillouin zone of the WZ phase in the thermal property calculations. The combined experimental and theoretical studies suggest that WZ InAs possesses volumetric heat capacity, weighted average phonon group velocity, average phonon-phonon scattering rate, and anharmonic scattering-limited thermal conductivity similar to those of the $\mathrm{ZB}$ phase. The comparable average phononphonon scattering rates may be attributed to the cancelation between additional acoustic-optical scattering processes and reduced coherence in the Fourier transform of different bonds in the WZ unit cell of an increased size compared to the ZB phase. The different thermal conductivity values observed in the InAs NWs of different phases are mainly caused by the different diameter and diffuse surface scattering mean free paths of the NWs. These findings provide insights that are useful for further studies of thermal transport in complex III-V NW structures with mixed $\mathrm{ZB}$ and $\mathrm{WZ}$ phases as well as sawtooth facets, ${ }^{67}$ which are designed to further reduce the lattice thermal conductivity. ${ }^{68}$

\section{ACKNOWLEDGMENTS}

The structure-characterization work of FZ and the theoretical modeling efforts of ALM at UT-Austin were supported by Office of Naval Research Award No. N00014-08-1-1168 and No. N000141-01-05-8-1. Part of this work was supported by the Nanometer Structure Consortium at Lund University (nmC@LU), the Swedish Foundation for Strategic Research (SSF), the Swedish Research Council (VR), and the Knut and Alice Wallenberg Foundation. The work of A.P. was supported by the Royal Physiographic Society in Lund and the Sweden-America Foundation. Density functional calculations for the phonon dispersions of $\mathrm{ZB}$ and $\mathrm{WZ}$ InAs were performed on the Intel Cluster at the Cornell Nanoscale Facility, part of the National Nanotechnology Infrastructure Network (NNIN) funded by the National Science Foundation. D.A.S. gratefully acknowledges Keith Refson for helpful discussions and access to his phonon branch sorting script, dispersion.pl. N.M. acknowledges ANR and the Nanosciences Foundation. The authors would like to thank Dr. Zhao Wang for sharing his work regarding the boundary scattering mean free path for NWs with an equilateral triangle cross section.

\footnotetext{
*Author to whom all correspondence should be addressed: lishi@mail.utexas.edu

${ }^{1}$ O. Madelung, Semiconductors: Data Handbook (Springer, Berlin, 2004).

${ }^{2}$ X. Y. Gong, H. Kan, T. Makino, T. Yamaguchi, T. Nakatskasa, M. Kumagawa, N. L. Rowell, A. Wang, and R. Rinfret, Cryst. Res. Technol. 30, 603 (1995).
}

${ }^{3}$ R. V. Shenoi, R. S. Attaluri, A. Siroya, J. Shao, Y. D. Sharma, A. Stintz, T. E. Vandervelde, and S. Krishna, J. Vac. Sci. Technol. B 26, 1136 (2008).

${ }^{4}$ S. Mou, A. Petschke, Q. Lou, S. L. Chuang, J. V. Li, and C. J. Hill, Appl. Phys. Lett. 92, 153505 (2008).

${ }^{5}$ L. E. Froberg, W. Seifert, and J. Johansson, Phys. Rev. B 76, 153401 (2007). 
${ }^{6}$ A. I. Persson, L. E. Froberg, S. Jeppesen, M. T. Bjork, and L. Samuelson, J. Appl. Phys. 101, 034313 (2007).

${ }^{7}$ S. A. Dayeh, D. A. Susac, K. L. Kavanagh, E. T. Yu, and D. L. Wang, Adv. Funct. Mater. 19, 2102 (2009).

${ }^{8}$ M. S. Dresselhaus, G. Chen, M. Y. Tang, R. G. Yang, H. Lee, D. Z. Wang, Z. F. Ren, J. P. Fleurial, and P. Gogna, Adv. Mater. 19, 1043 (2007).

${ }^{9}$ Z. Zanolli, F. Fuchs, J. Furthmuller, U. von Barth, and F. Bechstedt, Phys. Rev. B 75, 245121 (2007).

${ }^{10}$ A. De and C. E. Pryor, Phys. Rev. B 81, 155210 (2010).

${ }^{11}$ M. T. Bjork, A. Fuhrer, A. E. Hansen, M. W. Larsson, L. E. Froberg, and L. Samuelson, Phys. Rev. B 72, 201307 (2005).

${ }^{12}$ C. Thelander, M. T. Bjork, M. W. Larsson, A. E. Hansen, L. R. Wallenberg, and L. Samuelson, Solid State Commun. 131, 573 (2004).

${ }^{13}$ D. B. Suyatin, C. Thelander, M. T. Bjork, I. Maximov, and L. Samuelson, Nanotechnology 18, 105307 (2007).

${ }^{14}$ C. Fasth, A. Fuhrer, L. Samuelson, V. N. Golovach, and D. Loss, Phys. Rev. Lett. 98, 266801 (2007).

${ }^{15}$ C. Thelander, K. A. Dick, M. T. Borgstrom, L. E. Froberg, P. Caroff, H. A. Nilsson, and L. Samuelson, Nanotechnology 21, 205703 (2010).

${ }^{16}$ A. I. Persson, Y. K. Koh, D. G. Cahill, L. Samuelson, and H. Linke, Nano Lett. 9, 4484 (2009).

${ }^{17}$ G. A. Slack, J. Phys. Chem. Solids 34, 321 (1973).

${ }^{18}$ G. A. Slack, J. Phys. Chem. Solids 48, 641 (1987).

${ }^{19}$ M. Roufosse and P. G. Klemens, Phys. Rev. B 7, 5379 (1973).

${ }^{20}$ A. Amith, I. Kudman, and E. F. Steigmeier, Phys. Rev. 138, A1270 (1965).

${ }^{21}$ E. F. Steigmeier and I. Kudman, Phys. Rev. 141, 767 (1966).

${ }^{22}$ E. F. Steigmeier and I. Kudman, Phys. Rev. 132, 508 (1963).

${ }^{23}$ D. J. Ecsedy and P. G. Klemens, Phys. Rev. B 15, 5957 (1977).

${ }^{24}$ H. M. Tutuncu and G. P. Srivastava, Phys. Rev. B 62, 5028 (2000).

${ }^{25}$ A. Al Shaikhi and G. P. Srivastava, Phys. Rev. B 76, 195205 (2007).

${ }^{26}$ H. Kitagawa, Y. Shibutani, and S. Ogata, Modell. Simul. Mater. Sci. Eng. 3, 521 (1995).

${ }^{27}$ D. Morelli, J. Heremans, C. Beetz, W. S. Woo, G. L. Harris, and C. Taylor, in Silicon Carbide and Related Materials, edited by M. G. Spencer, R. P. Devaty, J. A. Edmond, M. A. Khan, R. Kaplan and M. Rahman (Institute of Physics, Washington, D.C., 1993), p. 313.

${ }^{28}$ P. G. Neudeck, J. Electron. Mater. 24, 283 (1995).

${ }^{29} \mathrm{~K}$. Jarrendahl and R. F. Davis, in SiC Materials and Devices, edited by Y.-S. Park (Academic, San Diego, 1998), Vol. 52, p. 13.

${ }^{30}$ G. A. Slack, J. Appl. Phys. 35, 3460 (1964).

${ }^{31}$ K. A. Dick, C. Thelander, L. Samuelson, and P. Caroff, Nano Lett. 10, 3494 (2010).

${ }^{32}$ L. Shi, D. Y. Li, C. H. Yu, W. Y. Jang, D. Kim, Z. Yao, P. Kim, and A. Majumdar, J. Heat Transf. 125, 881 (2003).

${ }^{33}$ F. Zhou, A. L. Moore, M. T. Pettes, Y. Lee, J. H. Seol, Q. L. Ye, L. Rabenberg, and L. Shi, J. Phys. D 43, 025406 (2010).

${ }^{34}$ K. A. Dick, P. Caroff, J. Bolinsson, M. E. Messing, J. Johansson, K. Deppert, L. R. Wallenberg, and L. Samuelson, Semicond. Sci. Technol. 25, 024009 (2010).

${ }^{35}$ G. Hollinger, R. Skheyta-Kabbani, and M. Gendry, Phys. Rev. B 49, 11159 (1994).
${ }^{36}$ D. G. Cahill, S. K. Watson, and R. O. Pohl, Phys. Rev. B 46, 6131 (1992).

${ }^{37}$ A. J. Bullen, K. E. O'Hara, and D. G. Cahill, J. Appl. Phys. 88, 6317 (2000).

${ }^{38}$ M. Nishio, S. Sawaya, S. Akita, and Y. Nakayama, J. Vac. Sci. Technol. B 23, 1975 (2005).

${ }^{39}$ F. P. Incropera and D. P. DeWitt, Fundamentals of Heat and Mass Transfer (Wiley, New York, 2007).

${ }^{40}$ R. Bowers, R. W. Ure, J. E. Bauerle, and A. J. Cornish, J. Appl. Phys. 30, 930 (1959).

${ }^{41}$ G. LeGuillou and H. J. Albany, Phys. Rev. B 5, 2301 (1972).

${ }^{42}$ J. Callaway, Phys. Rev. 113, 1046 (1959).

${ }^{43}$ D. T. Morelli, J. P. Heremans, and G. A. Slack, Phys. Rev. B 66, 195304 (2002).

${ }^{44}$ S. Baroni, S. D. Gironcoli, A. D. Corso, and P. Giannozzi, Rev. Mod. Phys. 73, 515 (2001).

${ }^{45}$ P. Giannozzi et al., J. Phys. Condens. Matter 21, 395502 (2009).

${ }^{46}$ G. B. Bachelet, D. R. Hamann, and M. Schluter, Phys. Rev. B 26, 4199 (1982).

${ }^{47}$ [http://www.quantum-espresso.org/pseudo.php].

${ }^{48}$ N. S. Orlova, Phys. Status Solidi B 119, 541 (1983).

${ }^{49}$ K. Refson, [http://www.tcm.phy.cam.ac.uk/castep/Phonons_Guide/ Castep_Phonons.html] (2009).

${ }^{50}$ S. O. Mariager, D. Khakhulin, H. T. Lemke, K. S. Kjaer, L. Guerin, L. Nuccio, C. B. Sorenson, M. M. Nielsen, and R. Feidenhans, Nano Lett. 10, 2461 (2010).

${ }^{51}$ U. Piesbergen, Z. Naturforsch. 18a, 141 (1963).

${ }^{52}$ I. Barin, O. Knacke, and O. Kubaschewski, Thermochemical Properties of Inorganic Substances (Springer, Berlin, 1977).

${ }^{53}$ A. Al Shaikhi and G. P. Srivastava, Phys. Status Solidi C 3, 1495 (2006).

${ }^{54}$ G. Chen, Nanoscale Energy Transport and Conversion (Oxford University Press, New York, 2005).

${ }^{55}$ P. G. Klemens, Proc. Phys. Soc. Sect. A 68, 1113 (1955).

${ }^{56}$ M. G. Holland, Phys. Rev. 132, 2461 (1963).

${ }^{57}$ G. A. Slack and S. Galginaitis, Phys. Rev. 133, A253 (1964).

${ }^{58}$ J. M. Ziman, Electrons and Phonons (Clarendon, Oxford, 1962).

${ }^{59} \mathrm{Z}$. Wang and N. Mingo (unpublished).

${ }^{60}$ A. K. McCurdy, H. J. Maris, and C. Elbaum, Phys. Rev. B 2, 4077 (1970).

${ }^{61}$ S. Barman and G. P. Srivastava, Appl. Phys. Lett. 81, 3395 (2002).

${ }^{62}$ P. G. Klemens, Phys. Rev. 148, 845 (1966).

${ }^{63}$ D. A. Broido, M. Malorny, G. Birner, N. Mingo, and D. A. Stewart, Appl. Phys. Lett. 91, 231922 (2007).

${ }^{64}$ A. Ward, D. A. Broido, D. A. Stewart, and G. Deinzer, Phys. Rev. B 80, 125203 (2009).

${ }^{65}$ M. A. Nusimovici and J. L. Birman, Phys. Rev. 156, 925 (1967).

${ }^{66}$ D. T. Morelli and G. A. Slack, in High Thermal Conductivity Materials, edited by S. L. Shinde and J. S. Goela (Springer, New York, 2006).

${ }^{67}$ P. Caroff, K. A. Dick, J. Johansson, M. E. Messing, K. Deppert, and L. Samuelson, Nature Nanotech. 4, 50 (2009).

${ }^{68}$ A. L. Moore, S. K. Saha, R. S. Prasher, and L. Shi, Appl. Phys. Lett. 93, 083112 (2008). 